\title{
A gestão do conhecimento holística: análise de aderência do modelo de Futami
}

Fábio Corrêa

Doutor e Mestre em Sistemas de Informação e Gestão do Conhecimento. MBA em Engenharia de Software e Governança de Tecnologia da Informação e Graduação em Sistemas de Informação. fabiocontact@gmail.com

Fabrício Ziviani

Doutor em Ciência da Informação pela UFMG . Professor Adjunto I do Programa de Pós-Graduação em Sistemas de Informação e Gestão do Conhecimento da Universidade FUMEC. Professor Adjunto VI da Universidade do Estado de Minas Gerais (UEMG)

fabricio.ziviani@fumec.br

Leandro Cearenço Lima

Mestre em Sistemas de Informação e Gestão do Conhecimento pela Universidade FUMEC, Especialização em Marketing Estratégico pela Universidade .Consultor de negócios no Itaú-Unibanco S/A.

leandrolima.panamericano@gmail.com

Maria Zuleila Carmona Regino Campos

Profissional com Mestrado em Administração pela PUC-MG , MBA Empresarial pela Fundação Dom Cabral Aposentada pela Companhia Energética de Minas Gerais (CEMIG)

zuleila@gmail.com

\section{Editor Científico: José Edson Lara}

Organização Comitê Científico

Double Blind Review pelo SEER/OJS

Recebido em 11.06.2018

Aprovado em 18.02.2019

Este trabalho foi licenciado com uma Licença Creative Commons - Atribuição - Não Comercial 3.0 Brasil 


\title{
Resumo
}

Objetivo do estudo: Esta pesquisa analisa a aderência do modelo de Futami à Gestão do Conhecimento holística.

Metodologia / abordagem: A Análise de Conteúdo, de cunho qualitativo, e a quantificação percentílica foram utilizados como procedimentos metodológicos, caracterizando a abordagem quali-quanti para a condução desta investigação.

Originalidade / relevância: $\mathrm{O}$ ajustamento da Gestão do Conhecimento ao paradigma holístico é frequentemente assinalado como um caminho para o desenvolvimento de modelos de gerenciamento do conhecimento. Mediante a esse delineamento esta investigação torna-se relevante por evidenciar se a proposta de Futami supriu a lacuna de um modelo holístico para essa forma de gestão.

Principais resultados: $\mathrm{O}$ modelo analisado contempla parcialmente os preceitos do holísmo no gerenciamento do conhecimento.

Contribuições teóricas / metodológicas: Esta pesquisa contribui por anunciar a permanência da lacuna existente, por ajustar o paradigma holístico ao gerenciamento do conhecimento e por delinear um meio para análise de modelos frente a Gestão do Conhecimento holística.

Palavras-chave: Gestão do conhecimento; Paradigma holístico; Gestão do conhecimento holística; Futami; Análise de conteúdo.

\section{The holistic knowledge management: adherence analysis of Futami model}

\begin{abstract}
Objective of the study: This research analyzes the adherence of the Futami model to holistic Knowledge Management.

Methodology / approach: Qualitative Content Analysis and percentile quantification were used as methodological procedures, characterizing the quali-quanti approach to conduct this research.

Originality / relevance: Adjusting Knowledge Management to the holistic paradigm is often marked as a pathway to the development of knowledge management models. Through this design, this investigation becomes relevant because it shows whether Futami's proposal filled the gap of a holistic model for this form of management.
\end{abstract}

Main results: The model analyzed partially contemplates the precepts of holism in knowledge management. 
Theoretical / Methodological Contributions: This research contributes by announcing the permanence of the existing gap, by adjusting the holistic paradigm to knowledge management and by outlining a means for model analysis against holistic Knowledge Management.

Keywords: Knowledge management; Holistic paradigm; Holistic knowledge management; Futami; Content analysis.

\section{La gestión del conocimiento holístico: análisis de adherencia del modelo de Futami}

\section{Resumen}

Objetivo del estudio: Esta investigación analiza la adherencia del modelo de Futami a la Gestión del Conocimiento holística.

Metodología / enfoque: El análisis de contenido cualitativo y la cuantificación porcentual se utilizaron como procedimientos metodológicos, caracterizando el enfoque cualitativo para realizar esta investigación.

Originalidad / relevancia: Ajustar la gestión del conocimiento al paradigma holístico a menudo se marca como un camino hacia el desarrollo de modelos de gestión del conocimiento. A través de este diseño, esta investigación se vuelve relevante porque muestra si la propuesta de Futami llenó el vacío de un modelo holístico para esta forma de gestión.

Resultados principales: El modelo analizado contempla parcialmente los preceptos del holismo en la gestión del conocimiento.

Contribuciones teóricas / metodológicas: Esta investigación contribuye al anunciar la permanencia de la brecha existente, al ajustar el paradigma holístico a la gestión del conocimiento y al describir un medio para el análisis de modelos contra la gestión holística del conocimiento.

Palabras Clave: Gestión del conocimiento; Paradigma holístico; Gestión del conocimiento holístico; Futami; Análisis de contenido.

\section{Introdução}

A Gestão do Conhecimento (GC) é um campo de pesquisa transversal que apresenta estudos diversificados (Del Massa, Damian \& Valentim, 2018; Tavares, Costa \& Leite, 2018; Nogueira \& Miranda, 2018), dentre os quais tem-se os modelos de gerenciamento do conhecimento, fundamentados no intento de aplicar a perspectiva teórica da GC na prática organizacional. No entanto, a GC é forjada no paradigma newtoniano-cartesiano e, portanto, os modelos de gerenciamento do conhecimento (re)apresentam os preceitos do reducionismo. 
Isso não é exclusividade da GC, pois o campo da Administração, que exprime as bases da gestão, é oriundo das influencias de Descartes e Newton (Silva, 2013; Andrade \& Amboni, 2017), assim como a Ciência da Computação, que apresenta os algoritmos e a modularização de software, sob o alicerce de dividir para conquistar (Ziviani, 2014; Cormen, 2017). A área da Saúde também se estabelece no paradigma reducionista (Rocha \& Monteiro, 2018; Antunes, Lagranha, Sousa, Silva \& Fraga, 2018; Copetti \& Folmer, 2018), o que evidencia o quanto este padrão científico influenciou - e ainda influencia - as ciências.

Especificamente na GC, os estudos de Holsapple e Joshi (1999), Rubenstein-Montano et al. (2001), Heisig (2009) e Fteimi (2015) anunciam que um caminho para o desenvolvimento de modelos de gerenciamento do conhecimento é o paradigma holístico, proposto como uma alternativa para tratar os problemas científicos não solvidos pelo paradigma anterior e ainda reinante: o reducionismo (Crema, 2015; Folloni, 2016; Pereira, 2017; Ramalho, Locatelli \& Silva, 2018).

Mediante a essa contundente (re)afirmação de que a GC deve ser pautada no anúncios do paradigma holístico, esta pesquisa delineia uma teoria que ajusta essas duas vertentes, intitulando-a de GC holística, e exprime as partes que devem ser consideradas perante aos princípios desse paradigma. Esse feito tem por intento subsidiar o objetivo desta pesquisa de analisar a aderência do modelo de Futami (2001) à GC holística. Assim, busca-se identificar se esse modelo supriu a lacuna de um modelo holístico para o gerenciamento do conhecimento.

De modo a organizar o exposto por esta pesquisa, a mesma é dividida em partes. Além desta introdução, a parte seguinte (seção 2) apresenta o referencial teórico que ajusta a GC ao paradigma holístico e delineia suas partes. Mediante a essa base teórica, os procedimentos metodológicos (seção 3) utilizados para a análise do modelo de Futami (2001), bem como a justificativa de exame deste modelo, são apresentados. A seguir (seção 3), o modelo é analisado e os resultados, advindos deste exame, são apresentados. Por conseguinte, as considerações finais são tecidas e as referências bibliográficas utilizadas finalizam esta pesquisa.

\section{Referência Teórico}

Para desenvolver uma teoria torna-se necessário revelar os aspectos teóricos-conceituais que a fundamenta. Portanto, esta seção discorre sobre a gênese da GC holística (subseção 2.1) e anuncia as dimensões (partes) que a constitui (subseção 2.2). 


\subsection{A Gestão do Conhecimento Holística}

Segundo Kuhn (1998), um paradigma resulta em como as ciências utilizam instrumentos metodológicos para abordarem seus problemas que, quando não solvidos, acarretam na proposição de novos instrumentos e teorias, resultando na concepção de um novo paradigma. Assim, para entendimento do paradigma holístico e ajustamento desse com a GC, torna-se necessário compreender seu antecessor, o paradigma reducionista.

Quando René Descartes (1596-1650) propôs a árvore do conhecimento e concebeu o entendimento do homem enquanto máquina esse filósofo e matemático, detentor de uma mente analítica, anunciou um método fundamentado na razão, que promoveu as primeiras segmentações no âmbito da ciência. Posteriormente, Isaac Newton (1642-1727), filósofo, matemático e astrônomo, coroou o método divisor de Descartes, buscando compreender as causas e efeitos dos fenômenos da ciência por meio da mecanização, matematização e racionalidade (Maciel \& Silva, 2008; Ferreira, Cardoso, Corrêa \& França, 2009; Crema, 2015).

O legado de Descartes e Newton culminou em um método que instituiu um paradigma científico, denominado paradigma newtoniano-cartesiano, em homenagem a seus propositores, ou reducionismo, em referência a divisão. Em essência, Descartes e Newton propõem que um fenômeno seja dividido em menores partes e que essas sejam analisadas buscando seu entendimento de modo disjunto das demais. Uma vez entendidas estas partes isoladamente se atinge a compreensão do fenômeno de forma totalitária (Capra, 2000; Crema, 2015; Ramalho, Locatelli \& Silva, 2018).

No âmbito da GC são propostos modelos. Um modelo de GC é uma representação esquemática da realidade que visa promover a orquestração das dimensões (partes) que circundam essa forma de gestão. Tais modelos desenvolvem o campo científico do gerenciamento do conhecimento e são forjados no paradigma reducionista, pois abordam partes (dimensões) específicas da GC, como processos e atividades (Sedighi \& Zand, 2012), cultura organizacional (Valmohammadi, 2010) e tecnologia da informação (Kumar, Singh \& Haleem, 2015), fragmentando essa gestão em partes e as analisando de modo disjunto do todo.

A exemplo, o modelo de Sánchez e Ponjuán Dante (2016) aborda as dimensões tecnologia da informação e a cultura organizacional, enquanto a estrutura de Moscoso-Zea et al. (2016) contempla os processos e atividades da GC, além das dimensões abordadas por Sánchez e Ponjuán Dante (2016). Essas estruturas buscam compreender a GC de forma 
totalitária, mas conforme Kuhn (1998), é diante da insuficiência de um paradigma que outro emerge, sendo esta a proposta do paradigma holístico.

Como posto por Crema (2015), o paradigma reducionista se tornou insuficiente para contemplar os problemas da ciência moderna, pois esses são mais intricados que os existentes na época de Descartes e Newton, sendo o paradigma holístico uma alternativa para o desenvolvimento científico contemporâneo. No contexto da GC, o paradigma holístico é apontado por Holsapple e Joshi (1999), Rubenstein-Montano et al. (2001), Heisig (2009) e Fteimi (2015), mediante a análise de 270 modelos de gerenciamento do conhecimento, como uma alternativa para o desenvolvimento de modelos de GC holísticos.

O paradigma holístico busca complementar o reducionismo e se dissocia desse por um aspecto específico e expressivo. Assim como o reducionismo, o paradigma holístico admite a redução do fenômeno em partes. No entanto, este apregoa que as partes devem ser analisadas considerando as demais, pois assim as conexões entre esses segmentos são mantidas, o que permite compreender o fenômeno de forma integra (Crema, 2015; Folloni, 2016; Pereira, 2017). Deste modo, enquanto o reducionismo permite regressar ao fenômeno e compreendê-lo de forma totalitária, o paradigma holístico consente a compreensão do fenômeno em sua integralidade, pois mantêm as conexões adjacentes entre as partes seccionadas.

Assim, o ajustamento do paradigma holístico para com a GC anuncia que os modelos de gerenciamento do conhecimento devem contemplar todas as dimensões (partes) desta forma de gestão em conjunto, para que se possa analisá-las considerando suas conexões e compreender o fenômeno do gerenciamento do conhecimento de forma íntegra. Esta forma de gerenciamento, orientada ao conhecimento e ajustada ao paradigma holístico, é denominada, nesta pesquisa, por GC holística.

Todavia, para que as partes possam ser analisadas considerando as demais torna-se necessário identificar quais são as partes que constituem o todo, o hólus da GC. Em outros termos "Pascal já dizia: 'Só posso compreender um todo se conheço, especificamente, as partes, mas só posso compreender as partes se conhecer o todo" (Morin, 2005, p. 181). Estas dimensões (partes) que dão forma ao hólus a GC holística constituem o objeto de estudo da subseção seguinte desta pesquisa. 


\subsection{Dimensões da Gestão do Conhecimento Holística}

A busca de estabelecer os elementos que constituem o gerenciamento do conhecimento remonta o ano de 1997, quando Skyrme e Amidon (1997), na publicação The knowledge agenda, anunciam os Fatores Críticos de Sucesso da GC como sendo os fatores-chave para que o gerenciamento do conhecimento tenha êxito.

Se um fator é crítico para o sucesso de uma forma de gestão esse se torna um elemento preponderante a ser considerado, pois remente a referida gestão ao seu êxito. Portanto, tal fator se estabelece como uma dimensão - parte do todo - a ser considerada em modelos que ambicionem promover o gerenciamento do conhecimento organizacional. Assim, a junção destes fatores anuncia as dimensões (partes) críticas da GC holística que devem ser contempladas conjuntamente em modelos de gerenciamento do conhecimento.

Mediante a esse delineamento, foi realizada uma revisão sistemática da literatura com o objetivo de identificar as dimensões (partes) críticas para o sucesso da GC. A busca foi promovida na base SCOPUS as 07:05 de 12 de agosto de 2017, considerando os descritores "knowledge management" and "critical success factors", no título e sem delimitação temporal. A conclusão desta revisão resultou em 13 dimensões que constituem as partes críticas para o gerenciamento do conhecimento holístico, fundamentadas em estudo de 1997 a 2015, sendo:

a) Estratégia: a estratégia de GC deve ser alinhada a estratégia organizacional para apoiar os objetivos almejados e esse alinhamento deve ser clarificado aos demais membros da organização para que compreendam os objetivos almejados pela proposta de gerenciamento do conhecimento (Skyrme \& Amidon, 1997; Lin \& Lin, 2006; Gai \& Xu, 2009; Altaher, 2010; Sedighi \& Zand, 2012);

b) Liderança e suporte da alta administração: os líderes devem atuar como modelos para os demais membros e a alta administração deve prover suporte para a operacionalização da GC (Davenport, De Long \& Beers, 1998; Wong, 2005; Akhavan, Jafari \& Fathian, 2006; Arif \& Shalhoub, 2014; Kumar, Singh \& Haleem, 2015);

c) Equipe de gestão do conhecimento: consiste em estabelecer uma equipe de indivíduos com papéis, atividades e responsabilidades específicas, visando a promoção da GC na organização (Davenport, De Long \& Beers, 1998; Wong, 2005; 
Wong \& Aspinwall, 2005; Al-Mabrouk, 2006; Lin \& Lin, 2006; Gai \& Xu, 2009; Valmohammadi, 2010; Arif \& Shalhoub, 2014);

d) Recursos (financeiro, humano, material e tempo): a GC, como outras iniciativas, demanda de pessoas para a promoção e aplicação dos processos e atividades de conhecimento, investimentos para aquisição de sistemas tecnológicos, insumos materiais e tempo para a operacionalização da GC (Wong \& Aspinwall, 2005; Gai \& Xu, 2009; Abbaszadeh, Ebrahimi \& Fotouhi, 2010; Sedighi \& Zand, 2012; Kumar, Singh \& Haleem, 2015);

e) Processos e atividades: os processos de identificação, armazenamento, compartilhamento e criação, dentre outros, são o centro da GC e anunciam o que pode ser feito com o conhecimento, devendo serem integrados ao fluxo de trabalho dos indivíduos de modo claro, sistemático e estruturado (Skyrme \& Amidon, 1997; Akhavan, Jafari \& Fathian, 2006; Abbaszadeh, Ebrahimi \& Fotouhi, 2010; Sedighi \& Zand, 2012);

f) Gestão de recursos humanos: os processos de recrutamento, desenvolvimento e retenção da gestão de recursos humanos são vitais para a GC por municiar a empresa de pessoas com conhecimentos relevantes ao propósito organizacional (Wong \& Aspinwall, 2005; Wong, 2005; Al-Mabrouk, 2006; Abbaszadeh, Ebrahimi \& Fotouhi, 2010; Valmohammadi, 2010; Sedighi \& Zand, 2012; Anggia et al., 2013; Kumar, Singh \& Haleem, 2015);

g) Treinamento e educação: a GC possui vocabulário particular e ferramentas tecnológicas específicas. Assim, os funcionários devem ser treinados para utilizar as ferramentas voltadas para o conhecimento, bem como educados para quanto ao vocabulário e linguagem da GC para que compreendam seus fundamentos teóricos (Davenport, De Long \& Beers, 1998; Akhavan, Jafari \& Fathian, 2006; Gai \& Xu, 2009; Abbaszadeh, Ebrahimi \& Fotouhi, 2010);

h) Motivação: os indivíduos precisam de estarem motivados para atuarem em prol da GC. Aspectos como recompensas, retorno financeiro e não financeiro, reconhecimento e valoração do indivíduos são meios considerados para a promoção da motivação pessoal (Davenport, De Long \& Beers, 1998; Lin \& Lin, 2006; Gai \& Xu, 2009; Sedighi \& Zand, 2012; Zieba \& Zieba, 2014); 
i) Trabalho em equipe: resulta na reunião de dois ou mais indivíduos que se influenciam e interagem em prol de um objetivo comum e que tendem a cooperação e elevação do potencial dos processos e atividades da GC, constituindo um meio fértil para a criação e compartilhamento do conhecimento (Wai, Hong \& Din, 2011; Sedighi \& Zand, 2012);

j) Cultura: Consiste em parâmetros de valores, normas e costumes sociais que moldam a forma como as pessoas se comportam. A GC demanda de uma cultura propícia que deve fomentar a partilha do conhecimento, admissão de erros, dentre outros aspectos que regem uma atmosfera voltada ao conhecimento. (Davenport, De Long \& Beers, 1998; Wong, 2005; Al-Mabrouk, 2006; Gai \& Xu, 2009; Valmohammadi, 2010);

k) Tecnologia da informação: a Tecnologia da informação é um facilitador eficaz de manipulação (captura, armazenamento e divulgação) do conhecimento, sendo um meio para o armazenamento e compartilhamento do conhecimento explícito e partilha do conhecimento tácito (Davenport, De Long \& Beers, 1998; Al-Mabrouk, 2006; Sedighi \& Zand, 2012; Anggia et al., 2013; Arif \& Shalhoub, 2014; Kumar, Singh \& Haleem, 2015);

1) Mensuração: medir é necessário para que seja possível aferir o progresso da GC. Resulta em determinar indicadores para avaliar o desempenho da iniciativa de gerenciamento do conhecimento e realizar diagnósticos para mensurar as dimensões organizacionais, visando identificar lacunas a serem sanadas quanto a GC (Davenport; De Long; Beers, 1998; Akhavan, Jafari \& Fathian, 2006; Sedighi \& Zand, 2012; Arif \& Shalhoub, 2014; Kumar, Singh \& Haleem, 2015);

m) Projeto Piloto: antes de promover uma iniciativa de conhecimento em toda a organização projetos piloto devem ser considerados, visando obter lições apreendidas e melhores práticas em menor escala para posterior expansão em âmbito organizacional (Skyrme \& Amidon, 1997; Akhavan, Jafari \& Fathian, 2006).

As 13 dimensões, acima descritas, constituem as partes da GC holística que devem ser consideradas em conjunto em um modelo de gerenciamento do conhecimento, conforme perspectiva do paradigma holístico. Essas dimensões compõem as unidades de registro da análise do modelo de Futami (2001), conforme procedimentos metodológicos delineados na seção seguinte desta pesquisa. 


\section{Metodologia}

Os procedimentos metodológicos utilizados para analisar o modelo de Futami (2001) são caracterizados pela abordagem qualitativa-quantitativa, com aplicação do método de Análise de Conteúdo e quantificação percentílica, respectivamente.

A Análise de Conteúdo consiste em um método de exame sistemático e descritivo do conteúdo das comunicações que permite a inferência de conhecimentos (Bardin, 1977), tendo por insumo textos escritos e passíveis de serem manipulados para atingimento do intento de uma pesquisa (Gaskell \& Bauer, 2002). Para aplicação deste método Bardin (1977) recomenda o estabelecimento das unidades de registro, que consiste nos elementos a serem investigados durante o exame de um conteúdo. No contexto desta pesquisa, as unidades de registros são as 13 dimensões da GC holística, definidas na subseção 1.2 desta pesquisa.

Deste modo, esta pesquisa aplica a Análise de Conteúdo, de cunho qualitativo, para identificar a presença ou ausência das 13 dimensões da GC holística no modelo de Futami (2001). Ciente de que a abordagem qualitativa admite a subjetividade do pesquisador (Minayo, 1998), o que demanda rigor científico em sua aplicação para manutenção dos resultados apreendidos (Flick, 2004), esta pesquisa faz uso costumeiro de citações diretas. Assim, as inferências de presença ou ausência das dimensões da GC holística serão compreendidas e o leitor poderá regressar ao conteúdo original do modelo analisado, provendo manutenibilidade aos resultados desta pesquisa e o rigor científico mister para a ciência.

Mediante a identificação das dimensões da GC no modelo a abordagem quantitativa é empregada. Esta abordagem fundamenta-se na quantificação e se vale do uso de técnicas matemáticas (Minayo, 1998). Deste modo, o cruzamento quantitativo entre as dimensões presentes e ausentes no modelo de Futami (2001) permite apreender o percentil de adesão desta estrutura à GC holística, resultando em uma resposta ao objetivo desta pesquisa.

A escolha de analisar o modelo de Futami (2001) é pautada em dois aspectos, sendo: i) campo científico de desenvolvimento; e ii) tipo de pesquisa. Em relação ao campo científico de desenvolvimento (i), o referido modelo foi construído no âmbito de um programa de Engenharia de Produção, área na qual são propostos diversos estudos de GC, como os de Terra (2005), Silva (2011), Luz (2017) e Gaeversen (2018). Assim, é uma área prolífica que tende a apresentar avanços científicos que contribuem para o desenvolvimento GC. 
Em relação ao tipo de pesquisa (ii), o modelo de Futami (2001) é uma pesquisa aplicada, na qual a estrutura desenvolvida foi posta em prática em um setor empresarial do segmento de eletroeletrônicos de bens de consumo. Deste modo, o estudo alia a perspectiva teórica e prática da GC e tende a trazer contribuições para a GC holística.

Ademais, a Revista Gestão \& Tecnologia (http://revistagt.fpl.edu.br/get/about/ editorialPolicies\#focusAndScope, recuperado em 03, novembro, 2008), para a qual este artigo é direcionado, assinala que em "seu escopo e prioridades concernem às pesquisas aplicadas, sejam descritivas, experimentais ou quase-experimentais". Portanto, a análise do referido modelo tende a promover avanços quanto a GC holística na perspectiva teórica e prática do conhecimento organizacional.

Por fim, ressalta-se que os resultados desta pesquisa não visam criticar ou enaltecer o modelo examinado. O objetivo é analisar a aderência desta estrutura à GC holística para identificar se esse modelo aborda o gerenciamento do conhecimento perante aos preceitos do paradigma holístico, revelando se uma proposta com esta envergadura foi atingida. Este o pilar único desta pesquisa, não havendo intento de diminuir ou elevar a pesquisa científica de outrem.

\section{Apresentação e Resultados}

Esta seção se segmenta em duas subseções. Na primeira (subseção 4.1) é apresentada a Análise de Conteúdo realizada sobre o modelo de Futami (2001), de modo a prover compreensão quanto as inferências realizadas sobre a existência das 13 dimensões da GC holística. Por conseguinte (subseção 4.2) são apresentados os resultados advindos desta análise e o percentil de adesão do referido modelo ao gerenciamento do conhecimento pautado no paradigma holístico.

\subsection{Apresentação da Análise do Modelo de Futami (2001)}

O modelo de Futami (2001) se apresenta como uma fusão das abordagens de Sistema de Trabalho de Alto Desempenho (STAD) (Nadler \& Gerstein, 1994), espiral do conhecimento (Nonaka \& Takeuchi, 1997) e times virtuais (Lipnack \& Stamps, 1999). Dessa fusão emerge o modelo (Figura 1) que visa a constituição de uma "arquitetura organizacional baseada em redes, para alavancar o conhecimento como recurso competitivo" (Futami, 2001, p. 100). 


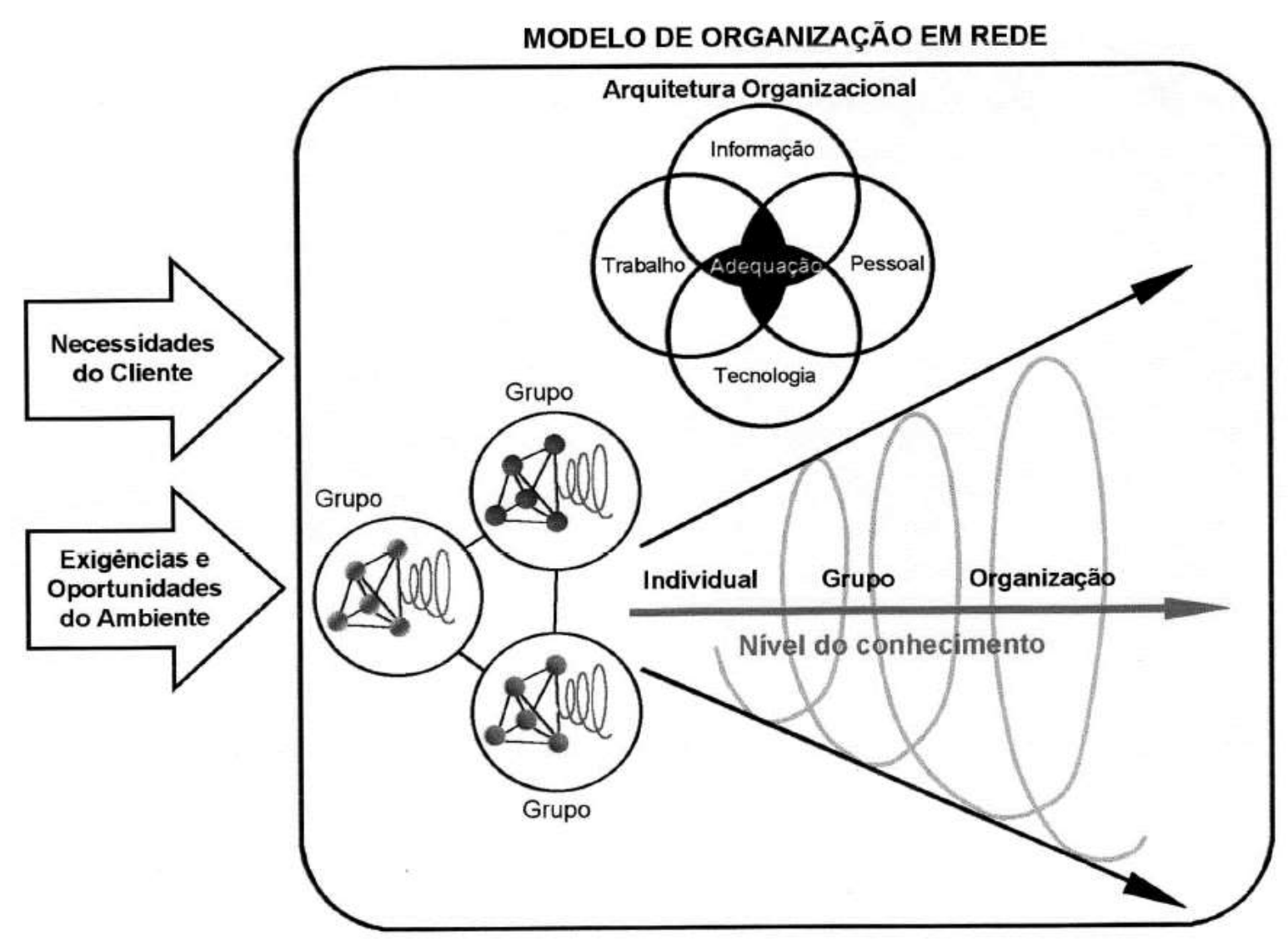

Figura 1: Modelo de Futami (2001).

Fonte: Adaptado de Futami (2001, p. 100).

A arquitetura organizacional é definida como "as maneiras pelas quais a empresa estrutura, coordena e administra o trabalho das pessoas em busca de objetivos estratégicos" (Futami, 2001, p. 48). Sobre esse prisma Futami (2001) propõe uma arquitetura organizacional na qual o trabalho dos indivíduos seja realizado em rede (times virtuais) de forma a permitir interações entre os conhecimentos dos indivíduos (tácito) e seu armazenamento (explícito) em um repositório de conhecimento, seguindo a abordagem STAD.

A abordagem STAD visa adequar informação, trabalho, pessoas e tecnologia de forma a atender as necessidades dos clientes e exigências e oportunidades do ambiente (Nadler \& Gerstein, 1994) e se fundamenta no trabalho em equipe. Assim, os grupos de pessoas promovem o trabalho em equipe para entregar produtos e serviços que atendam às necessidades dos clientes e exigências do ambiente (Futami, 2001). O modelo de Futami (2001) apregoa que esse trabalho seja realizado em redes virtuais segundo a abordagem de Lipnack e Stamps (1999), a qual demanda que as pessoas atuem sob um determinado propósito e estabeleçam conexões face a face ou por meios tecnológicos. 
Para Futami (2001) a abordagem STAD é uma "alternativa viável aos modelos tradicionais, como [...] administração científica e o modelo burocrático, que restringem o trabalho do conhecimento" (Futami, 2001, p. 101). As redes virtuais se apresentam como "um caminho mais fácil de realizar a transição da hierarquia/burocracia para a organização em rede" (Futami, 2001, p. 78). Assim, Futami (2001) defende que o STAD de Nadler e Gerstein (1994) e os times virtuais de Lipnack e Stamps (1999) oferecem uma alternativa de trabalho evolutiva no que tange as relações de comando da estrutura hierárquica, alta formalização e padronização dos processos atribuídos pela estrutura burocrática, significando que a STAD e os times virtuais são abordagens positivas para as tratativas voltadas ao conhecimento.

No que tange a espiral do conhecimento, Futami $(2001,145)$ defende que seu modelo “organiza o conhecimento explícito e cria uma forma de aproveitar melhor o conhecimento tácito das pessoas" e que "o sistema de trabalho em rede [referente ao próprio modelo] foi idealizado para impulsionar o processo de conversão em "espiral" do conhecimento" (Futami, 2001, p. 146). Para o autor:

\begin{abstract}
A equipe de trabalho organizado em rede será a unidade básica para gerar a dinâmica de criação do conhecimento organizacional. O objetivo principal é proporcionar um campo em que o conhecimento humano possa ser criado e expandido através da interação de forma a criar uma dinâmica entre o conhecimento tácito e o conhecimento explícito, conforme o modelo em espiral de Nonaka e Takeuchi (1997). (Futami, 2001, p. 107)
\end{abstract}

Para implantação de seu modelo Futami (2001) propõe 4 fases: 1) "Coleta e diagnóstico de dados"; 2) "Projeto do novo sistema de trabalho"; 3) "Implementação"; e 4) "Melhoria contínua" (Futami, 2001, p. 102).

A "coleta e diagnóstico de dados" (1) (Futami, 2001, p. 102) tem por intento avaliar a integração entre o sistema técnico (tecnologia e informação) e social (pessoas e trabalho) da organização. Essa fase é aderente a dimensão Mensuração da GC holística, uma vez que objetiva prover uma análise inicial do ambiente organizacional (diagnóstico) em prol do conhecimento para mensurar as dimensões organizacionais, visando identificar lacunas a serem sanadas quanto a GC (Akhavan, Jafari \& Fathian, 2006). Essa fase se se subdivide em quatro etapas: 1.1) "analisar a necessidade do cliente, do ambiente e as estratégias"; 1.2) "analisar o processo de trabalho"; 1.3) "analisar o sistema social"; e 1.4) "identificar as oportunidades da gestão do conhecimento" (Futami, 2001, p. 102). 
A subfase de "analisar a necessidade do cliente, do ambiente e as estratégias" (1.1) (Futami, 2001, p. 104) considera uma leitura do ambiente externo, pois a nova forma de trabalho em equipe deve ser regida pelas necessidades dos clientes, ambiente e estratégias organizacionais. Segundo Futami (2001) esta análise resulta na busca de três relações de estímulo, sendo: a) identificar os fatores ambientais que estimulam a estratégia; b) as estratégias que estimulam a organização; e c) a relação entre a estratégia e a vigente estrutura organizacional. As análises devem ser realizadas em prol do conhecimento de forma a permitir visualizar se a estrutura Futami é orientada ao conhecimento e a aprendizagem organizacional (FUTAMI, 2001).

A subfase seguinte, denominada "analisar o processo de trabalho" (1.2) (Futami, 2001, p. 104), almeja identificar como a estrutura da organização lida com o conhecimento nos processos de trabalho existentes. Futami (2001) assinala que o organograma da empresa é uma ferramenta pertinente para analisar as funções (cargos) versus o fluxo de trabalho e considera importante avaliar seis itens: a) estrutura formal; b) os elementos do sistema técnico; c) descrição do processo de trabalho; d) detalhamento do fluxo de trabalho; e) processos formais de trabalho; e f) tecnologias utilizadas nos processos de trabalho. Essas duas subfases (1.1 e 1.2) não se aderem a nenhuma das 13 dimensões da GC holística.

A subfase de "análise do sistema social" (1.3) (Futami, 2001, p. 105) visa analisar a cultura organizacional, conforme dimensão Cultura da GC holística. A cultura organizacional consiste em parâmetros de valores, normas e costumes sociais que moldam a forma como as pessoas se comportam (Al-Mabrouk, 2006). Para Futami (2001) o aspecto cultural é imperativo em relação ao conhecimento tácito e devem ser avaliados sete itens, sendo: padrões de comunicação, modos de interação dos indivíduos, estruturas de grupo, valores organizacionais, práticas informais do trabalho, padrões de liderança informal e normas informais do trabalho.

A última subfase do diagnóstico resulta em "identificar as oportunidades da gestão do conhecimento" (1.4) (Futami, 2001, p. 106). Mediante as análises anteriores, fundamentadas no modelo STAD de Nadler \& Gerstein (1994), Futami (2001) insere esta subfase como uma etapa de consolidação do diagnóstico sob o aspecto do conhecimento. A ideia é que sejam identificadas oportunidades de melhoria quanto a criação do conhecimento organizacional, sendo essa identificação voltada para a apreensão de fatores inibidores (barreiras) à aprendizagem organizacional (Futami, 2001). 
Ao findar do diagnóstico (1) se inicia o "projeto do novo sistema de trabalho" (2) (Futami, 2001, p. 106) com o intuito de projetar uma nova forma de trabalho em equipe organizada em rede, voltada para a produção e entrega de produtos e serviços que atendam às exigências externas analisadas na fase anterior. Segundo Futami (2001) essa fase cria um campo para a interação virtual, subsidiado pela tecnologia - aderente a dimensão Tecnologia da informação da GC holística -, permitindo a ocorrência dos modos de conversão entre o conhecimento tácito e implícito, advindos de Nonaka e Takeuchi (1997).

Visando promover nexo para que o trabalho em equipe seja produtivo, Futami (2001) faz uso da abordagem de times virtuais proposta por Lipnack e Stamps (1999), considerando os aspectos propósito, pessoas e conexões, sendo subdividida em cinco etapas: 2.1) "definir um propósito"; 2.2) "definir a unidade de trabalho (grupo)"; 2.3) "definir as conexões (pessoas e processos de trabalho)"; 2.4) "construir uma base de conhecimento (memória organizacional)"; e 2.5) "definir a tecnologia a ser utilizada" (Futami, 2001, p. 107).

A subfase "definir um propósito" (2.1) (Futami, 2001, p. 108) consiste em determinar, claramente, o objetivo que culminou na criação da rede de forma a dar subsídio ao grupo para administrar a nova estrutura e o processo de trabalho. "Definir a unidade de trabalho (grupo)" (2.2) (Futami, 2001, p. 108) é constituir a equipe de trabalho por meio da seleção das pessoas. A subfase de "definir as conexões (pessoas e processos de trabalho)" (2.3) (Futami, 2001, p. 108) é orientada a acoplar as pessoas com os processos de trabalho, tendo por elo de ligação a informação.

Essas três subfases se relacionam com a dimensão Trabalho em equipe da GC holística, pois estabelecem um objetivo comum para a condução do trabalho (Sedighi \& Zand, 2012) e provê meio para criação e compartilhamento do conhecimento (Wai, Hong \& Din, 2011).

A subfase "construir uma base de conhecimento (memória organizacional)" (2.4) (Futami, 2001, p. 109) abarca a criação de repositório de conhecimento para melhoria do acesso e transferência do conhecimento na rede virtual. Por fim, "definir a tecnologia a ser utilizada" (2.5) (Futami, 2001, p. 108) resulta na determinação das tecnologias que apoiaram o novo processo de trabalho em times virtuais.

Ambas as subfases supracitadas (2.4 e 2.5) se aderem a dimensão Tecnologia da informação da GC holística, por posicionarem a tecnologia como um facilitador eficaz de manipulação (captura, armazenamento e divulgação) do conhecimento (Al-Mabrouk, 2006) e 
demarcam a organização do conhecimento explícito em repositórios (Davenport, De Long \& Beers, 1998; Arif \& Shalhoub, 2014; Kumar, Singh \& Haleem, 2015).

As fases anteriores, denominadas "Coleta e diagnóstico de dados" (1) e "Projeto do novo sistema de trabalho" (2) (Futami, 2001, p. 102), têm por intento planejar a novo sistema de trabalho, sendo a fase seguinte de "implementação" (3) (Futami, 2001, p. 110) destinada a operacionalizar o modelo, ou seja, colocar em prática o que foi planejado anteriormente. Por fim, a última fase apregoa a "melhoria contínua" (4) (Futami, 2001, p. 110), na qual o intento é avaliar a eficiência do novo sistema implementado e promover melhorias (Futami, 2001).

\subsection{Resultados da Análise do Modelo de Futami (2001)}

Evidenciando as qualidades do estudo, Futami (2001, p. 146) pondera que o modelo de organização em rede tem a "possibilidade de ser operacionalizado em paralelo com a estrutura formal da organização, podendo coexistir, formal ou informalmente, com a estrutura vigente". Assim, o modelo se apresenta como uma perspectiva para a condução do trabalho na organização e não com uma forma que seja assumida por essa, visando derrocar a estrutura préexistente.

Segundo o autor o modelo foi aplicado em uma "empresa do setor eletroeletrônico que fabrica bens de consumo da linha branca" (Futami, 2001, p. 112), especificamente na unidade de sistema de refrigeração. Os participantes consideraram que o novo sistema de trabalho, proposto pelo modelo, permite que as pessoas interajam e compartilhem seus conhecimentos tácitos, o que impulsiona a formação da competência na unidade do sistema de refrigeração.

Além supracitado feedback dos participantes, Futami (2001) assinala que esses indivíduos mencionaram que a rede de conhecimento, proporcionada pelo modelo, concede "visibilidade para todos os membros do grupo dos assuntos que estão sendo tratados" (Futami, 2001, p. 143). Segundo Futami (2001, p. 143) "Essa visibilidade permite que outras pessoas possam contribuir mais proativamente com seu conhecimento e lançar novas 'luzes' na resolução de problemas".

Ademais, o repositório de conhecimento explícito - vertente tecnológica do projeto permitiu uma melhor organização dos conhecimentos dos indivíduos que participaram da implementação do modelo (Futami, 2001). Todavia, os participantes sugeriram "um treinamento mais detalhado em relação à utilização do software e aos fundamentos teóricos envolvidos na concepção do trabalho" (Futami, 2001, p. 144). O autor apresenta o repositório 
de conhecimento, implementado no Lótus Notes da IBM, e suas funcionalidades, mas não assinala o treinamento dos indivíduos para o uso da ferramenta, bem como não prevê isso no modelo apresentado.

Esse aspecto corrobora com a necessidade apresentada pela dimensão Treinamento e educação da GC holística, pois os funcionários devem ser treinados para compreender a GC e utilizar as ferramentas voltadas para o conhecimento (Gai \& Xu, 2009; Abbaszadeh, Ebrahimi \& Fotouhi, 2010), bem como educados para quanto ao vocabulário e linguagem da GC (Davenport, De Long \& Beers, 1998; Al-Mabrouk, 2006; Akhavan, Jafari \& Fathian, 2006) para que compreendam seus fundamentos teóricos. Assim, a aplicação prática do modelo evidencia que a negligência desta dimensão resultou em um aspecto percebido pelos participantes da aplicação prática do modelo, legitimando que o treinamento e a educação é um fator crítico para o sucesso da GC holística.

Em essência o modelo de Futami (2001) propõe uma nova forma de trabalho que se estabelece pela constituição de redes virtuais, formada por grupos de indivíduos, que compartilham seus conhecimentos por meio de uma plataforma tecnológica. Os conhecimentos compartilhados, resultante das interações entre as pessoas, são armazenados junto aos conhecimentos existentes, como documentos e relatórios, em um repositório de conhecimento que constitui a memória organizacional.

De forma ampla o modelo promove a convergência das dimensões Trabalho em equipe e Tecnologia da informação da GC holística para a constituição de rede de trabalho voltada ao conhecimento. Assim, o modelo aborda a reunião de dois ou mais indivíduos que se influenciam e interagem (Wai, Hong \& Din, 2011) e que tendem a cooperação e elevação do potencial de processos e atividades da GC (Sedighi \& Zand, 2012), constituindo um meio fértil para a criação e compartilhamento do conhecimento (Wai, Hong \& Din, 2011). Tais interações são impulsionadas pela TI, sendo esse um meio pelo qual o conhecimento explícito tende a ser armazenado (Wong \& Aspinwall, 2005) e compartilhado na organização (Sedighi \& Zand, 2012; Anggia et al., 2013; Kumar, Singh \& Haleem, 2015).

Devido a orientação ao trabalho em equipe com ênfase na tecnologia e, embora seja considerado o fator cultural no modelo, esse aborda a GC descrevendo, especificamente, uma forma de promover o trabalho em times virtuais. Por síntese, o modelo apresenta os seguintes dimensões da GC holística. 
a) Estratégia: não identificado;

b) Liderança e suporte da alta administração: não identificado;

c) Equipe de gestão do conhecimento: não identificado;

d) Recursos (financeiro, humano, material e tempo): não identificado;

e) Processos e atividades: Sim. Coleta e diagnóstico de dados, projeto do novo sistema de trabalho, implementação e melhoria contínua (não diretamente relacionados a manipulação do conhecimento);

f) Gestão de recursos humanos: não identificado;

g) Treinamento e educação: não identificado;

h) Motivação: não identificado;

i) Trabalho em equipe: sim. Ponto central do modelo para a constituição de times virtuais;

j) Cultura: sim. Diagnóstico dos fatores culturais pela relevância desses em relação ao conhecimento tácito;

k) Tecnologia da informação: sim. Presente na totalidade do modelo para a constituição de times virtuais e repositórios de conhecimento;

1) Mensuração: sim. Promove a medição, por meio do diagnóstico, de fatores externos (necessidades do cliente e ambiente), estratégia, processos de trabalho e sistema social (cultura organizacional);

m) Projeto Piloto: não identificado.

Mediante a análise promovida sobre o modelo de Futami (2001) os fatores acima ponderados são dispostos na Figura 2, que exprime a adesão do referido modelo as dimensões da GC holística, concernentes a esta pesquisa. A presença de uma dimensão a posiciona na escala 1 (extremidade externa) do gráfico e sua ausência a assinala na escala 0 (centro). 


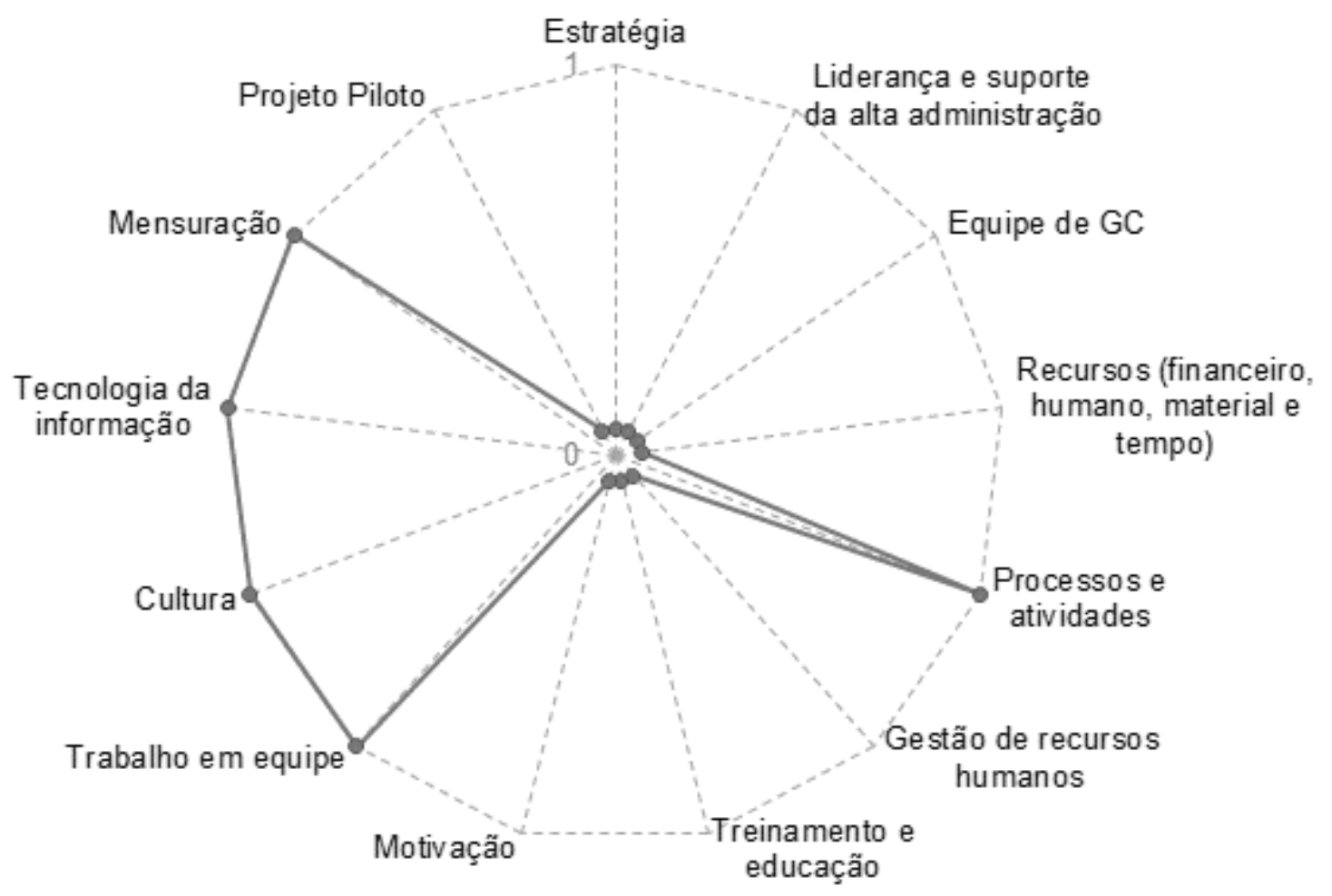

Figura 2:Adesão do modelo de Futami (2001) as dimensões da Gestão do Conhecimento holística.

Fonte: dados da pesquisa.

Assim, o modelo de Futami (2001) contempla as dimensões Processos e atividades, Trabalho em equipe, Cultura, Tecnologia da informação e Mensuração, o remetendo ao percentual de 38,5\% (5 do total de 13 dimensões) de adesão ao que considera por GC holística, conforme caracterização apresentada nesta pesquisa.

\section{Considerações Finais}

Esta pesquisa se alicerçou no intento de analisar a aderência do modelo de Futami (2001) à GC holística. Para atingimento desse objetivo, primeiramente a GC holística foi descrita e, posteriormente, as dimensões que a conforma foram evidenciadas. Mediante ao embasamento teórico apresentado o referido modelo foi analisado por meio do uso de abordagem mista (qualitativa-quantitativa).

Por resultado, tem-se que o modelo de Futami (2001) se adere em 38,5 pontos percentuais à GC holística. Isso revela que um modelo de gerenciamento do conhecimento fundamentado 
no paradigma holístico ainda não foi atingido, o que remete a necessidade de desenvolvimento de novas estruturas, considerando as dimensões anunciadas nesta pesquisa.

Ressalta-se que esse resultado não é conclusivo, pois esta pesquisa se limita por realizar a análise de um modelo datado de 2001. No entanto, o modelo examinado trata-se de uma estrutura proposta após a sinalização de Holsapple e Joshi (1999) e Rubenstein-Montano et al. (2001) quanto a necessidade de contemplar a GC mediante aos preceitos do paradigma holístico. Deste modo, a análise do referido modelo foi instigada pela possibilidade deste atingir uma abordagem holística do gerenciamento do conhecimento organizacional, tanto pelo aspecto teórico (acadêmico) quanto pela perspectiva prática (pesquisa aplicada).

Todavia, essa limitação acena oportunidades de pesquisas futuras, sendo a promoção da análise de outros modelos, seguindo os procedimentos metodológicos adotados nesta pesquisa,para que se possa obter uma ótica ampliada do quanto a GC tem caminhado para uma abordagem orientada ao paradigma holístico.

\section{Referências}

Abbaszadeh, M. A., Ebrahimi, M., \& Fotouhi, H (2010). Developing a causal model of critical success factors for knowledge management implementation. Anais do International Conference on Education and Management Technology, Cairo, Egito.

Akhavan, P., Jafari, M., \& Fathian, M. (2006). Critical success factors of knowledge management systems: a multi-case analysis. European Business Review, 18(2), 97-113.

Al-Mabrouk, K. (2006). Critical success factors affecting knowledge management adoption: a review of the literature. Anais do Innovations in Information Technology, Dubai, Emirados Árabes.

Altaher, A. M. (2010). Critical success factors of implementation knowledge management process. Anais do International Conference on Information Society, Londres, Inglaterra.

Andrade, R., \& Amboni, N. (2017). TGA - Teoria Geral da Administração. Elsevier: Brasil.

Anggia, P. et al (2013). Identifying critical success factors for knowledge management implementation in organization: a survey paper. Anais do International Conference on Advanced Computer Science and Information Systems, Bali, Indonésia.

Antunes, C. P. et al. (2018). Revisão sistemática sobre práticas corporais na perspectiva das práticas integrativas e complementares em saúde. Motrivivência, 30(55), 227-247.

Arif, M. J., \& Shalhoub, M. H. B. (2014). Critical success factors with its effective role in knowledge management initiatives in public and private organizations in Saudi Arabia: experts' perspectives. Life Science Journal, 11(6),636-645. 
Bardin, L. (1977). Análise de conteúdo. Lisboa: Edições 70.

Capra, F. A teia da vida: uma nova compreensão científica dos sistemas vivos. (9a ed.) São Paulo: Cultrix.

Copetti, J., Soares, R., \& Folmer, V. (2018). Educação e saúde no contexto escolar: compartilhando vivências, explorando possibilidades. (2a ed.) Uruguaiana: Universidade Federal do Pampa.

Cormen, T. H. (2002). Algoritmos: teoria e pratica. Rio de Janeiro: Campus.

Crema, R. (2015). Introdução à visão holística: breve relato de viagem do velho ao novo paradigma. (6a ed.) São Paulo: Summus.

Davenport, T. H., De Long, D. W., \& Beers, M. C. (1998). Successful knowledge management projects. Sloan Management Review, 39(2), 43-57.

Del Massa, H. C. O., Damian, I. P. M., \& Valentim, M. L. P. (2018). Competência em informação no apoio à Gestão do Conhecimento. Informação \& Sociedade, 28(1), 257-267.

Ferreira, V. C. P. et al (2009). Modelos de gestão. (3a ed.) Rio de Janeiro: Editora FGV.

Flick, U. (2004). Uma introdução à pesquisa qualitativa. (2a ed.) Porto Alegre: Bookman.

Folloni, A. (2016). Introdução à teoria da complexidade. Curitiba: Jaruá Editora.

Fteimi, N. (2015). Analyzing the literature on knowledge management frameworks: Towards a normative knowledge management clas-sification schema. Anais do 23rd European Conference on Information Systems, Münster, Alemanha.

Futami, A. H. (2001). Um modelo de Gestão do Conhecimento para a melhoria de qualidade do produto. Dissertação de mestrado, Universidade Federal de Santa Catarina, Florianópolis, $\mathrm{SC}$, Brasil.

Gaeversen, L. (2018). Compartilhamento de conhecimento nas atividades de gestão da Escola de Engenharia da UFRGS. Dissertação de mestrado, Universidade Federal do Rio Grande do Sul, Rio Grande do Sul, RS, Brasil.

Gai, S.; Xu, C (2009). Research of critical success factors for implementing knowledge management in China. Anais do International Conference on Information Management, Innovation Management and Industrial Engineering, Xi'an, China.

Gaskell, G., \& Bauer, M. W. (2002). Pesquisa qualitativa com texto, imagem e som. Petrópolis: Vozes.

Heisig, P. (2009). Harmonisation of knowledge management - comparing $160 \mathrm{KM}$ frameworks around the globe. Journal of Knowledge Management, 13(4), 4-31. 
Holsapple, C. W., \& Joshi, K. D. (1999). Description and analysis of ex-isting knowledge management frameworks. Anais do 32nd Annual Hawaii International Conference on IEEE, Maui, Estados Unidos.

Kuhn, T. S. (1998). A estrutura das revoluções científicas. (5a ed.) São Paulo: Editora Perspectiva.

Kumar, S., Singh, V., \& Haleem, A. (2015). Critical success factors of knowledge management: modelling and comparison using various techniques. International Journal of Industrial and Systems Engineering, 21(2), 180-206.

Kumar, S., Singh, V., \& Haleem, A. (2015). Critical success factors of knowledge management: modelling and comparison using various techniques. International Journal of Industrial and Systems Engineering, 21(2), 180-206.

Lin, Y-C., \& Lin, L-K. (2006). Critical success factors for knowledge management studies in construction. Anais do International Symposium on Robotics and Automation in Construction, Tóquio, Japão.

Lipnack, J., \& Stamps, J. (1999). Virtual teams; the new way to work. IEEE Engineering Management Review, 27(4), 90-95.

Luz, A. A. (2017). Um modelo para gestão de transferência de conhecimento e tecnologia para estágio acadêmico. Tese de doutorado, Universidade Tecnológica Federal do Paraná, Ponta Grossa, PR, Brasil.

Maciel, C., \& Silva, A. (2008). Gerenciando pessoas utilizando modelos holísticos. Revista da Administração Contemporânea, 12(1), 35-58.

Minayo, M. C. S. (1998). O desafio do conhecimento: pesquisa qualitativa em saúde. (5a ed.) São Paulo: Hucitec.

Morin, E. (2005). Ciência com consciência. (8a ed.) Rio de Janeiro: Bertrand Brasil.

Nadler, D. A., \& Gerstein, M. S. (1994). Projetos de sistemas de trabalho de alto desempenho: como organizar pessoal, trabalho, tecnologia e informação. In: Nadler, D. A., Gerstein, M. S., \& Shaw, R. B. Arquitetura organizacional: a chave para a mudança empresarial (pp. 95-114). Rio de Janeiro: Campus.

Naghavi, M., Dastaviz, A., \& Nezakati, H. (2013). Relationships among critical success factors of knowledge management and organizational performance. Journal of Applied Sciences, 13(5), 755-759.

Nogueira, A. P., \& Miranda, A. C. D. (2018). Gestão do conhecimento no setor público: um estudo sobre os artigos publicados em periódicos nacionais no período 2005-2015. Encontros Bibli, 23(52), 73-83.

Nonaka, I., \& Takeuchi, H. (1997). Criação de conhecimento na empresa: como as empresas japonesas geram a dinâmica da inovação. (10a ed.) Rio de Janeiro: Campus. 
Pereira, M. F. (2017). A gestão organizacional em busca do comportamento holístico. In: Angeloni, M. T. Organizações do conhecimento: infra-estrutura, pessoas e tecnologia (pp. 2-28). São Paulo: Saraiva.

Ramalho, W., Locatelli, R. L., \& Silva, S. D. C. D. (2018). Análise organizacional sob a ótica da teoria da complexidade: proposição e aplicação de um modelo. Revista Gestão \& Tecnologia, 18(2), 200-223.

Rocha, J. R., \& Monteiro, L. V. B. (2018). A dimensão espiritual na compreensão do fenômeno saúde-doença na psicologia da saúde. Caderno de Graduação, Ciências Biológicas e da Saúde, 4(2), 15-30.

Rubenstein-Montano, B. et al (2001). A systems thinking framework for knowledge management. Decision Support Systems, 31(1), 5-16.

Sedighi, M., \& Zand, F. (2012). Knowledge management: review of the critical Success factors and development of a conceptual classification model. Anais do International Conference on ICT and Knowledge Engineering, Bankok, Tailândia.

Sedighi, M.; Zand, F. (2012). Knowledge management: Review of the Critical Success Factors and development of a conceptual classification model. Anais do International Conference on ICT and Knowledge Engineering, Bangkok, Thailand.

Silva, R. O. (2013). Teorias da administração. (2a ed.) São Paulo: Pearson Education do Brasil.

Silva, R. S. G. (2011). Proposta de diretrizes para o desenvolvimento da gestão do conhecimento por meio de comunidades de prática. Dissertação de mestrado, Universidade de São Paulo, São Paulo, SP, Brasil.

Skyrme, D., \& Amidon, D. (1997). The knowledge agenda. Journal of Knowledge Management, 1(1), 27-37.

Tavares, R. B., Costa, S. M. D. S., \& Leite, F. C. L. (orgs.) (2018). Comunicação da informação, gestão da informação e gestão do conhecimento. Brasília: Ibict.

Terra, J. C. C. (2005). Gestão do conhecimento: o grande desafio empresarial. (5a ed.) Rio de Janeiro: Elsevier.

Valmohammadi C. (2010). Investigation and assessment of critical success factors of knowledge management implementation in Iranian small-to-medium sized enterprises. Journal of Applied Sciences, 10(19), 2290-2296.

Valmohammadi, C. (2010). Investigation and assessment of critical success factors of knowledge management implementation in Iranian small-to-medium sized enterprises. Journal of Applied Sciences, 10 (19), 2290-2296. 
Wai, Y. M.; Hong, A. N. H., \& Din, S. (2011). B. Critical success factors and perceived benefits of knowledge management implementation: Towards a conceptual framework. Australian Journal of Basic and Applied Sciences, 5(10), 754-760

Wong, K. Y. (2005). Critical success factors for implementing knowledge management in small and medium enterprises. In-dustrial Management \& Data Systems, 105(3), 261-279.

Wong, K. Y., \& Aspinwall, E. (2005). An empirical study of the important factors for knowledge-management adoption in the SME Sector. Journal of Knowledge Management, 9(3), 64-82

Zieba, M., \& Zieba, K. (2014). Knowledge management critical success factors and the innovativeness of KIBS companies. Engineering Economics, 25(4), 458-465.

Ziviani, N. (2014). Projeto de algoritmos com implementações em pascal e c. (3a ed.) Cengage Learning. 